\title{
Valores expresivos de la puntuación en los mensajes instantáneos de WhatsApp
}

\section{Expressive values of punctuation in WhatsApp instant messages}

PALOMA SERRANO GARCÍA

Facultad de Filosofía y Letras, Universidad Autónoma de Madrid

palomaserranogar@gmail.com

https://orcid.org/0000-0002-2022-981X.

Recibido: 10/10/2019 Aceptado: 18/11/2019.

Cómo citar: Serrano García, Paloma (2019), "Valores expresivos de la puntuación en los mensajes instantáneos de WhatsApp”, Revista Estudios del Discurso Digital (REDD), REDD, 2019 (2): 34-59.

DOI: https://doi.org/10.24197/redd.2.2019.34-59

Resumen: En este trabajo se analiza el valor pragmático-discursivo de los signos de puntuación utilizados en WhatsApp a través de muestras de conversaciones reales. En primer lugar, se reflexiona sobre las cuestiones ortográficas y estilísticas que determinan el uso de la puntuación y se detallan las propuestas que se han hecho sobre la puntuación en las nuevas tecnologías, así como las condiciones de enunciación de los mensajes instantáneos en WhatsApp que pueden influir en la selección de la puntuación. Por último, se analizan los valores expresivos de la puntuación utilizada en WhatsApp teniendo en cuenta la variable de la edad. Partiendo de la premisa de que existe una búsqueda de la relevancia óptima (Sperber y Wilson, 1986), se trata de sistematizar los cambios que se están produciendo en la puntuación en WhatsApp.

Palabras clave: discurso digital; mensajería instantánea; puntuación; emoticonos; WhatsApp.

Abstract: This paper analyzes the pragmatic and discursive value of the punctuation marks used in WhatsApp through samples of real conversations. First, we reflect on the orthographic and the stylistic issues that determine the use of punctuation and we detail approaches have been made about punctuation in new technologies, as well as the conditions of enunciation of WhatsApp instant messages that can influence the punctuation selection. Finally, we analyze the expressive values of the punctuation used in WhatsApp considering the age variable. Starting from the premise that there is a search for optimal relevance (Sperber and Wilson, 1986), we try to systematize changes in WhatsApp punctuation.

* Agradezco a Rosario González Pérez el ser mi guía en la realización de esta investigación y ayudarme a mejorarla con todos sus comentarios y sugerencias. No obstante, todos los fallos que puedan encontrarse en este trabajo son responsabilidad mía. 
Keywords: digital discourse; instant messaging; punctuation; emoticons; WhatsApp.

Sumario: Introducción, La puntuación en el texto escrito tradicional, La puntuación en las nuevas tecnologías, Los mensajes instantáneos de WhatsApp, Metodología, Resultados, Discusión, Conclusiones.

Summary: Introduction, Punctuation in traditional written text, Punctuation in new technologies, WhatsApp instant messages, Methodology, Results, Discussion, Conclusions.

\section{INTRODUCCIÓN}

El estudio de la puntuación se ha abordado tradicionalmente desde un punto de vista normativo (ortografías y libros de estilo). Sin embargo, con la aparición de las nuevas tecnologías han comenzado a proliferar los usos no normativos de la lengua en general y de la puntuación en particular, por lo que resulta necesario un nuevo enfoque en su tratamiento. El objeto de estudio del presente trabajo es analizar el valor pragmático y discursivo de la puntuación en los mensajes instantáneos de WhatsApp siguiendo la metodología de la lingüística de corpus. Su originalidad e interés reside en que, aunque se suelen señalar algunos usos no normativos de la puntuación en los estudios sobre textos digitales, aún no se han realizado trabajos de campo pormenorizados que indaguen en las causas de estos usos y en los factores que influyen en su utilización en WhatsApp.

Se parte de la hipótesis de que en los mensajes instantáneos de WhatsApp la puntuación responde a una función retórico-emotiva, de tal forma que los usos no normativos son consecuencia de la búsqueda de la relevancia óptima en una interacción mediada por un dispositivo electrónico. Teniendo esto en cuenta, los objetivos que nos planteamos son los siguientes:

1) Examinar los valores que se le han atribuido a la puntuación en el texto escrito tradicional y las particularidades de los textos digitales que determinan la puntuación empleada.

2) Delimitar los cambios que se están produciendo en el valor pragmático y discursivo de la puntuación y su nueva normalización en los mensajes instantáneos de WhatsApp a través de muestras de uso reales.

3) Valorar la influencia de la edad de los hablantes en el uso de la puntuación en WhatsApp. 
Para delimitar estos objetivos, nos hemos planteado las siguientes preguntas de investigación:

a) ¿Qué cambios se están produciendo en las funciones de la puntuación?

b) ¿Existe una norma en el uso de la puntuación en los textos digitales?

c) ¿En qué medida afecta la edad al uso de la puntuación?

En el presente trabajo, en primer lugar, se reflexionará sobre las cuestiones ortográficas y estilísticas que determinan el uso de la puntuación. En segundo lugar, se detallarán cuáles son las propuestas que se han hecho sobre el uso de los signos de puntuación en las nuevas tecnologías, así como las condiciones de enunciación de los mensajes instantáneos en WhatsApp que puede influir en la selección de la puntuación. Por último, se realizará un análisis cualicuantitativo de la puntuación utilizada en WhatsApp teniendo en cuenta la variable edad.

\section{LA PUNTUACIÓN EN EL TEXTO ESCRITO TRADICIONAL}

\section{1. Perspectivas en el estudio de la puntuación}

\section{1. 1. Puntuación prosódica}

Puesto que la puntuación se origina como una transcripción de la entonación (Parkes, 1992), tradicionalmente se han considerado los signos de puntuación como elementos que transcriben las pausas en la oralidad y guían la lectura en voz alta. Esta concepción se mantiene aún hoy en autores como Gómez Torrego (2006: 77), Linares (1979: 31), Martínez de Sousa (2004: 458), Millán (2005: 20), Moliner (1967, s.v. puntuación) y Seco (2011, s.v. puntuación). La Real Academia Española (a partir de ahora RAE) también adoptó este criterio en la Ortografía de 1754 y así se ha mantenido hasta la última edición, de $2010^{1}$.

Sin embargo, numerosos autores defienden que actualmente la oralidad no tiene relación con la puntuación, sino que es un producto exclusivo de la escrituralidad (Cassany, 1993, 1995, 1999; Ferreiro,

\footnotetext{
1 Según la edición anterior, de 1999, con la puntuación "se pretende reproducir la entonación de la lengua oral” (RAE, 1999: 55). Así se mantuvo también en el Diccionario panhispánico de dudas (DPD, s.v. signos ortográficos), donde se dice que, entre otras funciones, sirven para marcar "las pausas y la entonación con que deben leerse los enunciados”.
} 
1996; Nunberg, 1990; Polo, 1974; Sánchez Iglesias, 2019)². A este respecto, Halliday (1990: 39) defiende que en la lectura en voz alta se tiende a introducir pausas en los límites gramaticales delimitados por la puntuación, pero en la oralidad también se realizan pausas en otros lugares que no se corresponden con una frontera gramatical, por ejemplo, cuando el hablante va a emitir una palabra inesperada por el destinatario. De igual forma, los signos de puntuación no tienen por qué representar una pausa ${ }^{3}$. Es más, para Halliday, el hecho de que se introduzcan pausas en la lectura en voz alta de un texto es simplemente una opción y es consecuencia de una traducción consciente del texto escrito a un medio oral $^{4}$.

\section{1. 2. Puntuación lógico-semántica}

En las lenguas romances, los signos de puntuación comenzaron siendo una adaptación de los signos latinos (Santiago, 1996). El desarrollo de la imprenta trajo consigo la unificación de los textos y la consolidación de la lectura silenciosa y, en consecuencia, en los siglos XVI y XVII, la puntuación lógico-semántica se fue imponiendo a la prosódica $^{5}$ (Santiago, 1998).

\footnotetext{
${ }^{2}$ Como explica Sánchez Iglesias, “[d]e la misma manera que la oralidad tiene sus componentes, o sus recursos, más o menos exclusivos, también los tiene lo escrito. Y un candidato perfecto, no cabe duda, para definir lo escrito es la puntuación” (2013: 141).

${ }^{3}$ El relacionar una pausa en la oralidad con la puntuación conlleva, por ejemplo, el típico error de colocar una coma entre el sujeto y el predicado, puesto que en la oralidad el hablante puede realizar una pausa en ese momento. También se puede dar el caso contrario: en un lugar donde hay un signo de puntuación no se produce ningún tipo de pausa. Esto es frecuente, por ejemplo, en las respuestas sí/no a preguntas, seguidas de vocativo, del tipo: “-Sí, señor” (pronunciado síseñor).

${ }^{4}$ En la actualidad, los textos escritos están destinados a la lectura silenciosa. Sin embargo, un texto escrito puede ser oralizado. En este sentido, los signos de puntuación ayudan también en la lectura en voz alta, aunque esta no es su principal función. La relación de los signos de puntuación con la melodía de la frase y la interpretación entonativa de los mismos al oralizar un texto es estudiada con detalle por Alcoba (2000).

${ }^{5}$ Así lo reflejaba Nebrija: "Lo mismo que en la lengua hablada es necesario realizar ciertas pausas distintivas, para que el oyente perciba las distintas partes de la frase y para que el locutor, una vez recuperado el aliento, hable con mayor energía así, en la escritura, hemos de hacer lo mismo para resolver ambigüedades, por medio de los signos de puntuación” (apud Millán, 2005: 20).
} 
Según la perspectiva lógico-semántica, la puntuación establece relaciones sintácticas y lógicas entre las distintas partes del discurso y determina el sentido del texto, eliminando posibles ambigüedades. Nunberg (1990: 17) define la puntuación como “a set of nonalphanumeric characters that are used to provide information about structural relations among elements of a text, including commas, semicolons, colons, periods, parentheses, quotation marks and so forth”6 ${ }^{\text {. }}$

Destaca esta visión de la puntuación por ser la postura que mantiene actualmente la RAE. En el proemio ortográfico del Diccionario de autoridades (1726), la Academia ya defendía la función lógicosemántica, y esta perspectiva la mantuvo en la primera ortografía de $1741^{7}$. Sin embargo, la RAE optó posteriormente por una postura prosódica que ha desterrado en la última edición, de $2010^{8}$.

\section{1. 3. Puntuación cohesiva}

En el acto de escritura, el escritor debe ser capaz de prever cómo interpretará el texto el lector para transmitir adecuadamente lo que desea comunicar. Teniendo esto en cuenta, los signos de puntuación constituyen elementos cohesivos que ofrecen instrucciones de procesamiento, que indican al lector las relaciones pragmáticodiscursivas existentes entre las distintas unidades ${ }^{9}$. Así pues, la puntuación actúa de forma similar a los marcadores del discurso

\footnotetext{
${ }^{6}$ Polo (1974: 292) o Serafini (1992: 242) también defienden esta como la única función de la puntuación. Sin embargo, llama la atención que otros autores que defendían la función prosódica de la puntuación, también tienen en cuenta el valor semántico de estos signos. Véase, por ejemplo, Martínez de Sousa (2004: 462), Millán (2005: 20) o Seco (2011, s.v. puntuación).

7 "Las claúsulas se dividen de varias maneras, y con varios signos, que no solo indican la division de la claúsula; sino el sentido de ella” (RAE, 1741: 261).

${ }^{8}$ En la OLE de 2010 se pone claramente de manifiesto que no se comparte ya la visión prosódica. Actualmente para la RAE, "no puede hablarse en rigor de que la puntuación reproduzca las propiedades prosódicas de los enunciados” (OLE, 2010: 287).

${ }^{9}$ En este sentido, es común la comparación de los signos de puntuación con las señales de tráfico: son instrucciones de circulación colocadas intencionalmente para dirigir la circulación del lector por el texto. Figueras (2001:48), por ejemplo, compara el uso del punto y aparte con el del stop, el de los dos puntos con el de una señal de fin de autovía, el de los guiones largos o el de los paréntesis con el de desvío obligatorio, el de los puntos suspensivos con el de carretera cortada, etc.
} 
(Sánchez Iglesias, 2013: 154) ${ }^{10}$. Esta es la visión defendida por Cassany (1987: 184, 1993: 11,1999: 30 y 31), Ferreiro (1996: 154), Figueras (2001: 32) y Sánchez Iglesias (2013: 153).

\section{2. Función demarcativa y unidades del sistema de puntuación español}

A pesar de las distintas perspectivas ofrecidas por diversos autores sobre las funciones de la puntuación, todos ellos coinciden en concebir la puntuación como un elemento que delimita unidades. De esta forma, si se asume que todos los signos de puntuación comparten esta función, se ve reforzada la idea de la puntuación como sistema.

De hecho, los autores determinan el inventario de signos de puntuación a partir de su función demarcativa. Así, dependiendo de las unidades delimitadas por el punto, Ferreiro (1996: 130) y Cassany (1999: 5) se plantean si este signo es un único puntema o tres diferentes: el punto y seguido, el punto y aparte y el punto final. Linares (1979), por su parte, no solo los diferencia, sino que desdobla la coma, el punto y coma, los dos puntos y el punto en catorce signos distintos ${ }^{11}$. Por otro lado, Benito Lobo (1992) incluye entre los signos de puntuación los espacios en blanco y las mayúsculas ${ }^{12}$.

\footnotetext{
${ }^{10}$ De hecho, también es común el paralelismo entre los marcadores del discurso y las señales de tráfico. Así lo expresa Montolío (2001: 21): “de la misma manera que las señales viarias indican itinerarios del tipo "curva peligrosa a la derecha”, "atención: cambio de rasante”, o “dirección obligatoria”, las instrucciones que aportan los elementos que estamos analizando son del tipo: "la información que viene a continuación constituye la causa de la que se deduce la que vendrá detrás", "la información que sigue se opone en el razonamiento argumentativo a la anterior”, o "atención: ahora llega la conclusión y, por tanto, la información que realmente interesa”.

${ }^{11}$ Por ejemplo, propone que existen tres comas distintas: coma-1 (para separar ideas con significados analógicos), coma-2 (es doble y delimita un inciso), coma-3 (marca elisiones, delimita vocativos y elimina ambigüedades) (Linares, 1979: 61-72).

${ }^{12}$ Catach (1980) también reflexiona sobre el papel de los espacios en blanco en relación con el concepto de elemento gráfico: “que veut dire «élément graphique»? En l'abscence d'un signe de ponctuation, que reste-t-il? Un blanc, lequel est déjà un signe, le plus primitif et essentiel de tous, un «signe en négatif»" (1980:18). Es más, como muestra Cassany (1999: 6), el espacio en blanco ofrece información determinante sobre las relaciones entre las unidades gráficas:
}

(i) a. Dibuja a las amarillas [a las chicas amarillas]

b. Dibuja alas amarillas [las extremidades superiores de las aves] 
Figueras (2001: 35 y 36), de acuerdo con la función demarcativa, divide los signos de puntuación en dos regímenes (cuadros 1 y 2):

\begin{tabular}{|c|c|c|}
\hline MARCADOR & UNIDAD DELIMITADA & \\
\hline Coma & $\begin{array}{c}\text { Sintagma } \\
\text { Dos puntos }\end{array}$ & $\begin{array}{c}\text { Nivel } \\
\text { Punto y coma }\end{array}$ \\
Punto y seguido & Enunciados oracionales \\
& Eláusula textual \\
Enunciado textual & \\
\hline Punto y aparte & Párrafo & Nivel \\
Punto final & Texto & macroestructural \\
\hline
\end{tabular}

Cuadro 1. Signos de primer régimen (Figueras, 2001: 35)

\begin{tabular}{|c|c|}
\hline $\begin{array}{c}\text { Introducción de un segundo } \\
\text { discurso }\end{array}$ & Marcadores de modalidad \\
\hline Guiones largos & Signos de interrogación \\
Paréntesis & Signos de exclamación \\
Comillas & Puntos suspensivos \\
\hline
\end{tabular}

Cuadro 2. Signos de segundo régimen (Figueras, 2001: 36)

Llegados a este punto, cabe plantearse si considerar que la puntuación delimita unidades no incurre en definiciones tautológicas. Por ejemplo: un punto delimita una oración y una oración es aquello que va entre puntos ${ }^{13}$. Por tanto, la cuestión reside en si es la puntuación la que determina la unidad o es la unidad la que selecciona la puntuación (Sánchez Iglesias, 2013: 150).

\section{3. Puntuación estilística}

Tradicionalmente, la puntuación ha sido tratada como una parte más de la ortografía. Sin embargo, a pesar de que los autores tratan de establecer una lista que concrete los usos de cada signo, pueden darse

En cuanto a las mayúsculas, Ferreiro (1996: 130) señala que "una de las pocas reglas verdaderamente normativas que hay en el uso actual de la puntuación se refiere a las mayúsculas, obligatorias después del punto así como al inicio del texto”.

13 Así explica Cassany (1987: 65) los problemas con los que se encuentran muchos estudiantes al aprender a puntuar siguiendo este tipo de reglas: "pedimos a los alumnos que pongan un punto al final de cada oración y cuando preguntan: "¿Qué es una oración?», más tarde o más temprano acabamos por responder: «Es aquello que hay entre punto y punto»”. 
variaciones que no se consideran un error: "puntuar un texto siempre tendrá mucho de arte, de toma de decisiones con frecuencia sutiles, y en caso de duda no habrá diccionario ni programa de ordenador que pueda darnos la solución correcta” (Millán, 2005: 12).

En efecto, la puntuación depende de lo que el escritor tenga la intención de comunicar a su lector en función de la situación comunicativa. De hecho, la adecuación a cada contexto está determinada por la búsqueda de la relevancia óptima (Sperber y Wilson, 1986) y es lo que permite que la puntuación adquiera nuevas funciones pragmáticas en los textos digitales. Así pues, existiendo normas que afectan directamente a la interpretación procedimental de la frase (1), el escritor puede seleccionar en ciertos casos un signo u otro para presentar la misma información de distinta forma (2).

(1) a. El gato que estaba sentado en el tejado maullaba mucho.

b. El gato, que estaba sentado en el tejado, maullaba mucho.

(2) a. Por fin había llegado; había unos ojos que la miraban de forma penetrante.

b. Por fin había llegado. Había unos ojos que la miraban de forma penetrante.

En los ejemplos de (1), el uso de la coma es determinante para obtener la interpretación de la oración. Así, en (1a) se está identificando al gato, mientras que en (1b) se ofrece información adicional acerca de ese gato. Esta coma permite distinguir sintácticamente entre una oración de relativo especificativa y una explicativa. Por el contrario, en (2) se ofrece la misma información, pero se presenta de distinta forma, es decir, en (2a) la información se encuentra más integrada que en (2b).

\section{LA PUNTUACIÓN EN LAS NUEVAS TECNOLOGÍAS}

Las tecnologías de la información y la comunicación están cambiando la forma de comunicarnos. Los términos netspeak (Crystal, 2005, 2005), ciberlenguaje, ciberhabla, lenguaje de internet o lenguaje electrónico se han acuñado para hacer referencia a la nueva variedad lingüística a la que el ciberespacio ha dado lugar. 
El ciberlenguaje se caracteriza por desvincular la comunicación del canal, desdibujando los límites entre oralidad y escritura ${ }^{14}$ (Baron, 2010; Cassany, 2006, 2012; Crystal, 2002, 2005; Figueras, 2014; Penas, 2018; Yus, 2001, 2010). La comunicación mediada por ordenador (a partir de ahora CMO) utiliza el sistema gráfico propio de la escritura y no permite la interacción cara a cara ni la riqueza prosódica de la oralidad. Sin embargo, esta escritura guarda relación con las características de emisión de la oralidad, como la falta de planificación, la informalidad, la interactividad o la espontaneidad. Así pues, no se puede asociar el ciberlenguaje con uno de estos dos extremos, sino que es una nueva variante que se nutre de ambos en función de una serie de parámetros (Mancera Rueda, 2016: 1481) y que ofrece nuevas posibilidades propias del medio electrónico (Crystal, 2005: 100).

Para suplir la ausencia de la prosodia y del paralenguaje ${ }^{15}$, se utilizan recursos tipográficos como las mayúsculas, los signos de puntuación, la escritura ideofonemática (Torres i Vilatarsana, 2003) o los emoticonos $^{16}$. Los usos no normativos de la puntuación en la CMO responden a un tipo de puntuación emergente que Figueras (2014) ha denominado retórico-emotiva y que surge de la competencia para generar textos relevantes (en términos de Sperber y Wilson, 1986) ${ }^{17}$. A través de elementos paralingüísticos, como marcas tipográficas, emoticonos, presencia/ausencia de puntuación o repetición de signos de segundo orden, los usuarios de la red tratan de suplir las carencias contextuales de este tipo de interacciones mediadas por un dispositivo electrónico.

14 En este sentido, Yus (2001, 2010) denomina estos discursos textos escritos oralizados.

${ }^{15}$ Es su uso también han podido influir otros factores propios de la comunicación digital, como el incremento exponencial de interlocutores y documentos y la ausencia de filtros o controles, dos de los cambios drásticos con los que Cassany (2012: 50-58) caracteriza la escritura en línea en contraposición con la lectura en papel.

${ }^{16}$ Los textos que utilizan este tipo de recursos para expresar la afectividad y las emociones han sido denominados por Jaffe et al. (1995) emotextos.

${ }^{17}$ Que estos usos no sean normativos (véase RAE, 2018) no quiere decir que no exista una norma (aunque tácita) en su uso: "el contraste de normas entre una época y otra(s) [o entre las diferentes situaciones comunicativas] no debe llevarnos a cometer el error de juzgar que la mera comprobación de la existencia de otra clase de normas significa la ausencia de ellas” (1990: 52). No obstante, para Alcántara (2014) no es posible encontrar una norma: "Los signos de puntuación aparecen en los mensajes instantáneos de manera impredecible y que parece tener más que ver con las costumbres del interlocutor que con normas o con una segmentación prosódica intuitiva” (2014: 238). 
Los usos no normativos de la puntuación suponen un aumento del esfuerzo de procesamiento que se compensa por la obtención de implicaturas débiles (Yus, 2010: 198). En el siguiente ejemplo se muestra cómo, tras el enriquecimiento inferencial (3b), el signo de interrogación ayuda, por un lado, a guiar la explicatura de alto nivel en cuanto a la actitud proposicional del hablante (3c) y, por otro lado, a recuperar la intensidad de la misma generada por la repetición de este signo (3d):

(3) a. <nenita69> alguien quiere hablar conmigo???????? Que me mande un privado.

b. <nenita69> ¿ [Hay] alguien [conectado a este canal] [que quiera hablar conmigo]?

c. <nenita69> está preguntando si hay alguien [conectado a este canal] [que quiera hablar con ella]

d. <nenita69> está preguntando con insistencia si hay alguien [conectado a este canal] [que quiera hablar con ella] (ejemplo de Yus, 2010: 206)

Los signos de puntuación establecen los límites de las unidades sintáctico-semánticas del texto escrito y la relación entre ellas. Sin embargo, la sintaxis de la CMO no sigue las normas de la sintaxis estándar, sino que se presenta de forma fragmentada o "telegráfica" (Herring, 2019) ${ }^{18}$. Por tanto, los usos no normativos de la puntuación en la CMO están relacionados con la gramática-e (Herring, 2019) y con las características propias del ciberhabla (Crystal, 2002).

Por otro lado, la puntuación no normativa y los emoticonos en la CMO son elementos paralingüísticos en tanto que expresan emociones, indican sexo o edad, modifican los significados de las palabras o regulan la interacción (Figueras, 2014: 150), y ambos responden a una función metarrepresentativa de la actitud proposicional del emisor. Por tanto, los emoticonos pueden considerarse signos de puntuación ${ }^{19}$.

\footnotetext{
${ }^{18}$ Sobre la gramática de los textos digitales en español, cfr. Gómez Torrego (2001).

19 Markman y Oshima (2007), por ejemplo, también defienden que los emoticonos pueden adquirir la función de la puntuación y basan su propuesta en la tendencia a situar los emoticonos al final de un enunciado. Para Sampietro (2016a), por el contrario, los emoticonos no actúan como signos de puntuación, puesto que tienen funciones que no poseen los signos de puntuación, como la intensificación de ideas expresadas en el texto escrito, la expresión de la informalidad o el realce de la identidad grupal. Sin embargo, estas funciones propias de los emoticonos no impiden su uso como signos de
} 
En definitiva, la puntuación es un sistema vivo que está evolucionando y modificando sus elementos y sus funciones. De hecho, una de las características propias de los textos digitales es la de transformar elementos existentes y darles un uso distinto del original. Así pues, la puntuación adquiere la función de guiar la interpretación retórico-emotiva; de igual forma, los emoticonos adquieren el valor cohesivo de la puntuación. No obstante, la puntuación normativa sigue presente en estos medios y la selección de una puntuación u otra implica ofrecer una imagen concreta al interlocutor.

\section{LOS MENSAJES INSTANTÁNEOS DE WHATSAPP}

WhatsApp es una aplicación de mensajería instantánea diseñada para telefonía móvil por Jan Koum y Brian Acton que usan más de mil millones de personas en 180 países distintos ${ }^{20}$. Este género textual es sincrónico, es decir, hay casi simultaneidad entre la emisión y la recepción del mensaje (Cassany, 2012: 71), por lo que estos apenas cuentan con planificación. Además, las relaciones que se establecen, por lo general, son de proximidad.

Así pues, la accesibilidad al teclado en un teléfono móvil, el registro, la planificación o las relaciones interpersonales son parámetros que van a influir en la puntuación empleada en WhatsApp. Estos factores, que intervienen en el uso de la puntuación, pueden indicar el grado de formalidad de este género textual y su relación con la oralidad.

\section{Metodología}

Para analizar el valor pragmático-discursivo del sistema de puntuación español en los mensajes instantáneos de WhatsApp, se ha recopilado un corpus con 366 mensajes de grupos de WhatsApp (139 de

puntuación. Por un lado, los signos de puntuación en la puntuación retórico-emotiva también son indicadores de informalidad, su repetición supone la intensificación de lo expresado en el texto y marcan la pertenencia a un grupo social. Por otro lado, la mayoría de emoticonos parecen poder adquirir una función cohesiva (aunque no siempre lo hagan) de forma que, a la vez que demarcan una unidad, guían la lectura indicando las emociones del hablante de una forma aún más rica que los modalizadores.

20 "More than 1 billion people in over 180 countries use WhatsApp to stay in touch with friends and family, anytime and anywhere” (Disponible en: https://www.whatsapp.com/about/, consulta: 09/05/2019). 
grupos familiares, 110 de grupos de amigos mayores de 50 años y 117 de grupos de amigos jóvenes) con 35 hablantes diferentes, cuyas edades se comprenden entre los 18 y los 29 años o son mayores de 50. El interés de comparar estas dos franjas de edad reside en que los primeros son nativos digitales, mientras que los segundos son inmigrantes digitales (véase Prensky, 2001).

Debido al tratamiento de datos de carácter personal, nos ajustamos a lo dispuesto en la Ley Orgánica de Protección de Datos Personales y Garantía de los Derechos Digitales ${ }^{21}$. Se ha informado previamente a los participantes que han cedido sus conversaciones de WhatsApp sobre cómo se tratarían sus datos personales y estos han consentido en el uso de los mismos con fines científicos firmando un Consentimiento Informado. Todos los datos han sido anonimizados.

Los ejemplos que aquí se ofrecen proceden de este corpus y están referenciados de la siguiente forma: WhatsApp = WA + número de conversación + número de línea. Además, el rango de edad 18-29 queda reflejado mediante una $\mathrm{J}$ (de joven) y el rango +50 , mediante una $\mathrm{M}$ (de mayor). El sexo puede identificarse a través de una codificación del tipo $\mathrm{H}$ para los hombres y $\mathrm{M}$ para las mujeres seguido de un número en función del orden de intervención (H1, H2, M1, M2, etc.).

\section{RESUltados}

Los signos de puntuación se han dividido en cuatro grupos: los signos de primer orden (punto, coma, punto y coma y dos puntos), los modalizadores (interrogación, exclamación y puntos suspensivos), los introductores de un segundo discurso (paréntesis, rayas y comillas) y aquellos emoticonos que sean utilizados con función demarcativa. Desde un punto de vista cuantitativo, los signos de primer orden se mantienen como los más utilizados, pero su uso es mucho menor que en un texto escrito tradicional. Además, los modalizadores tienen una importante presencia. Por su parte, los emoticonos se utilizan en ocasiones como signos de puntuación y los introductores de un segundo discurso apenas se documentan.

21 Disponible en: https://boe.es/boe/dias/2018/12/06/pdfs/BOE-A-2018-16673.pdf, consulta: 03/05/2019. 


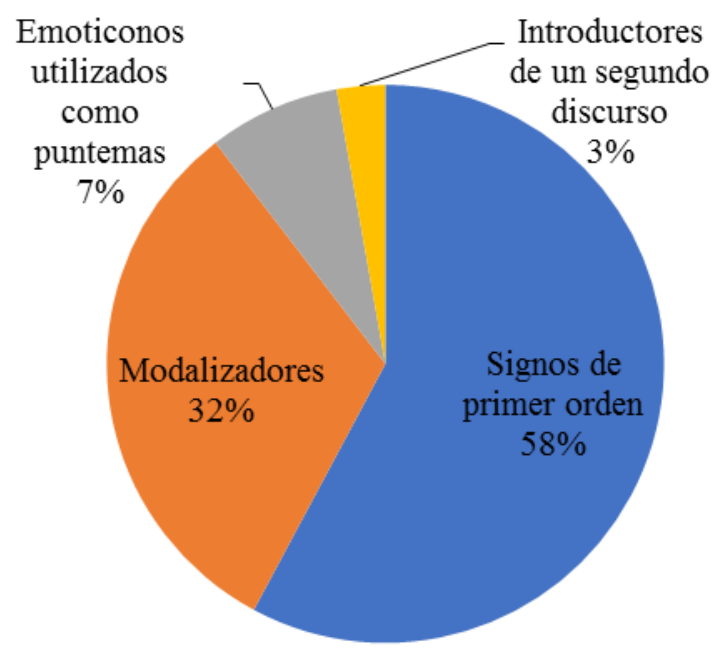

Figura 1. Signos de puntuación totales en función de cuatro grupos

Por lo que se refiere al uso en función de la edad, los jóvenes apenas utilizan puntuación en comparación con el 84\% de uso de la puntuación de los mayores de 50 años.

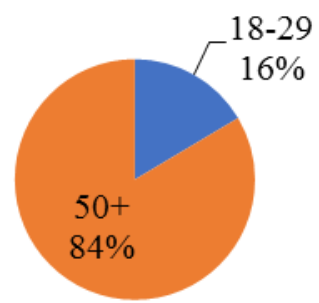

Figura 2. Signos de puntuación totales en función de la edad

Atendiendo a cada signo y en función de la edad, se mantienen los jóvenes como los hablantes que menos signos de puntuación utilizan en general y los signos que más usan son los modalizadores. Los hablantes de mayor edad, por el contrario, se muestran más conservadores en el uso de los signos de primer orden, a la par que utilizan los signos de puntuación con una función retórico-emotiva, como se observa por el uso abundante de los signos modalizadores y de los emoticonos como signos de puntuación. Por último, en cuanto a los introductores de un segundo discurso, solo se documenta el uso del paréntesis en los jóvenes y el paréntesis y las comillas en los mayores. 


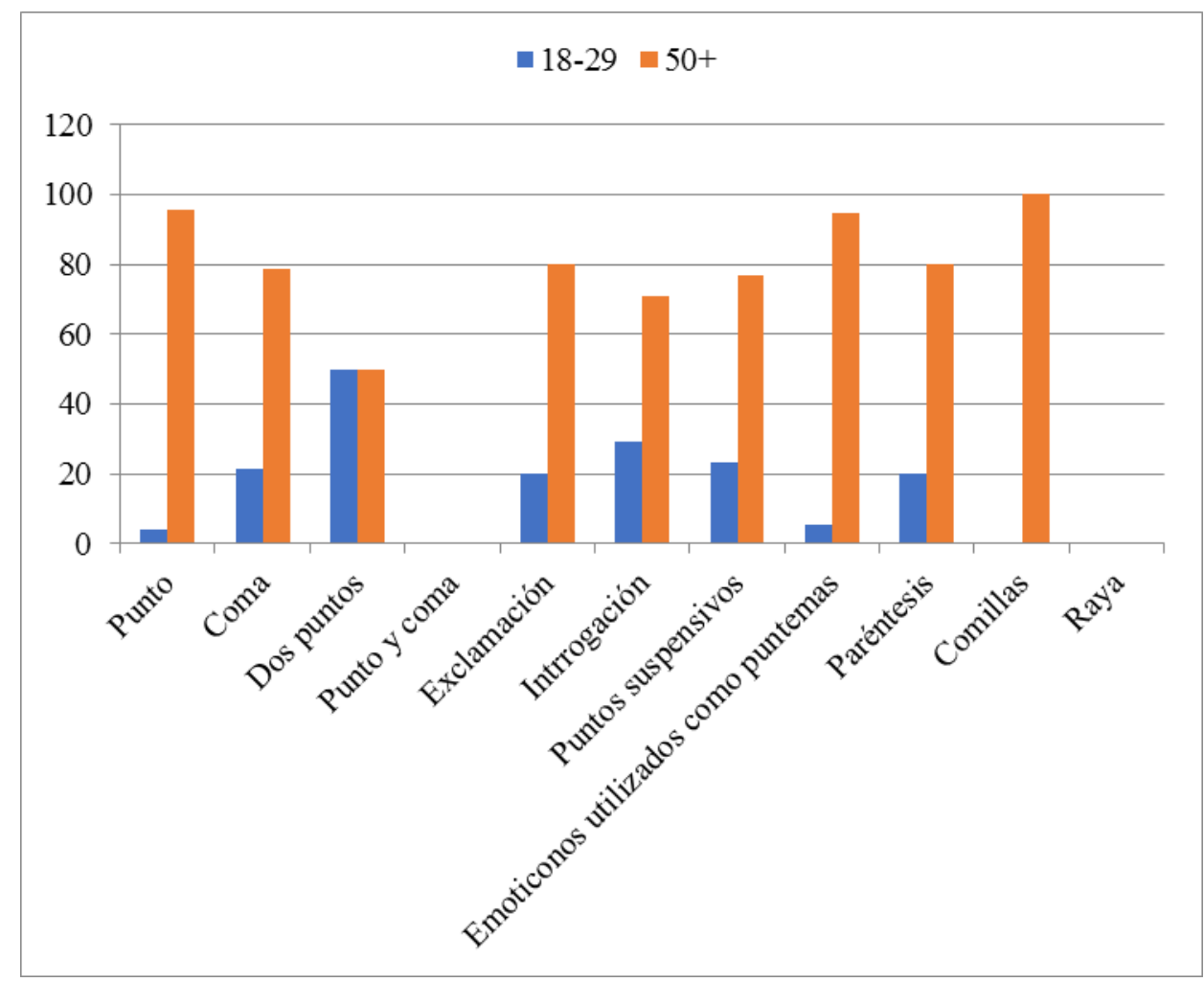

Figura 3. Uso de cada signo en función de la edad

Por lo que se refiere a la repetición de modalizadores, reflejo de los usos retórico-emotivos, pese a que los jóvenes utilizan menos signos, los que usan los repiten con más frecuencia que los mayores.

\begin{tabular}{|r|c|c|}
\cline { 2 - 3 } \multicolumn{1}{c|}{} & $18-29$ & $50+$ \\
\hline Repetición del signo de exclamación & $3 / 5$ & $6 / 20$ \\
& $60 \%$ & $30 \%$ \\
\hline Repetición del signo de interrogación & $3 / 12$ & $1 / 29$ \\
& $25 \%$ & $3.45 \%$ \\
\hline Más de tres puntos suspensivos & $2 / 3$ & $2 / 10$ \\
& $66.66 \%$ & $20 \%$ \\
\hline Total & $8 / 20$ & $9 / 59$ \\
& $40 \%$ & $15.25 \%$ \\
\hline
\end{tabular}

Tabla 1. Repetición de signos modalizadores

Por el contrario, los usos normativos del signo de apertura de interrogación solo se documentan en hablantes mayores. No obstante, no 
se utiliza ningún signo de apertura de exclamación y el de interrogación solo aparece en un $10 \%$ en los hablantes mayores.

\begin{tabular}{|r|c|c|}
\cline { 2 - 3 } \multicolumn{1}{c|}{} & $18-29$ & $50+$ \\
\hline Apertura de exclamación & $0 / 5$ & $0 / 20$ \\
& $0 \%$ & $0 \%$ \\
\hline Apertura de interrogación & $0 / 12$ & $3 / 29$ \\
& $0 \%$ & $10.34 \%$ \\
\hline Total & $0 / 17$ & $3 / 49$ \\
& $0 \%$ & $6.12 \%$ \\
\hline
\end{tabular}

Tabla 2. Signos de apertura de interrogación y de exclamación

\section{DisCUSIÓN}

\section{1. Signos de primer orden}

Se confirma la tendencia a la desaparición de los signos de primer orden. En primer lugar, llama la atención la ausencia del punto y coma en todo el corpus y la casi desaparición de los dos puntos, documentados únicamente en dos mensajes, que se limitan a introducir algunas enumeraciones (4a) o discursos reproducidos (4b), pero en otros casos son sustituidos por otros signos (4c).

(4) a. M2: te digo los días que no puedo: 27, 29, 4, 5 y 17 tengo seminarios, el 12 y 13 exposición (asi que mejor que el día de antes no tenga que ir) (WA02, 29-30, J)

b. M1: ....Nivel de conversación: - "buenos días" - "Serán pa ti" (WA06, 91-92, M)

c. M4: 2 buenas noticias, tú mejorando y a mi suegro le han dado el alta. (WA06, 29-30, M)

Por su parte, la coma y el punto siguen teniendo presencia en el corpus, aunque sus usos son generalmente no normativos y varían en función del hablante. La segmentación de la información en distintos mensajes se convierte en un método claro y visual que utilizan los hablantes para delimitar unidades. Así pues, el hecho de dividir los enunciados en varios mensajes implica el ahorro de puntemas (5).

(5) a. M5: Minimo 
M5: Jjjajajaja

M5: Yo hoy voy con la calma

M5: No voy a romperme (WA04, 14-17, J)

b. M1: Si la cosa fluye

M1: Volverán

M1: Jajajajajaj (WA05, 48-50, J)

Los mayores, sin embargo, tienden menos a fragmentar los enunciados en distintos mensajes. En algunos casos, envían toda la información que quieren comunicar en un solo mensaje más largo donde, no obstante, también se observa este estilo fragmentario. Así, por ejemplo, en (6) se hace un uso abundante del punto delimitando oraciones simples.

(6) M1: Eso digo yo. Pero es peor la Carmen, si el Alex está sobrio. Parece una gata. Siento que se pelean por mí. Qué stres. Ahora siesta (WA06, 100-101, M)

En este sentido, cuando no hay fragmentación solo se mantienen el punto y seguido (7) y la coma (8), pero el punto y aparte y el punto final se eliden.

(7) M2: A mi me han dicho que vaya a investigacion a ver si me lo pueden cambiar y no tengo que poner el papel, pero las de investigacion no han llegado aun. Las estoy esperando y mientras tanto voy a rellenar el ecpone y solicita pata adelantar. Espero no tener mucha cola luego (WA01, 40-43, J)

(8) M3: Acabo de hablar con el dueño, hoy está cerrado pero ya le he dicho que somos 13 e iremos a las 15 ,me ha dicho que le llame mañana para recordarselo pero que si tenemos sitio, quereis que le pregunte mañana sobre el menú? (WA07, 36-38, M)

En cuanto a la coma, no parece haber una tendencia clara en su uso. Esta tiende a aparecer con una función ilativa encadenando un enunciado tras otro en lugares donde la norma indica que deben usarse otros signos (8). Por el contrario, se encuentra completamente ausente para separar interjecciones repetidas (9). Sin embargo, para señalar elisiones (10) y 
para delimitar los vocativos (11) o incisos (12) hay más variación. Con todo, por lo general, los hablantes de mayor edad son más conservadores que los más jóvenes.

(9) H2: Ya lo sabemos jajajaja

M1: JAJAJAJAJAJAJ (WA05, 4-5, J)

(10) a. M1: Esa era la puerta de las chicas, a la azqda, los chicos (WA01, 5, M)

b. H1: Con Pablo 13. (WA07, 43, M)

(11) a. M2: Gracias, Eduardo (WA07, 22, M)

b. H1: Llama Andrea (WA05, 3, J)

(12) a. M1: Esta niña, como diría mi madre, hay que llevarla a algún sitio $O$ (WA06, 20, M)

b. M1: Y ver si el Skype de mi ordena q fue el tuyo funciona (WA03, 46, J)

Por tanto, los únicos signos de primer orden que se mantienen son el punto y la coma, pero estos se muestran como delimitadores de unidades independientemente de su naturaleza y de las relaciones que se establecen entre ellas. Por su parte, el punto y coma está totalmente ausente y los dos puntos, a pesar de documentarse, apenas tienen presencia. Es más, solo son utilizados en dos mensajes.

\section{2. Emoticonos utilizados como signos de puntuación}

Los emoticonos, en ciertos contextos, pueden adquirir la función cohesiva de la puntuación sustituyendo signos de puntuación de primer orden. De esta forma, a la vez que delimitan, guía la interpretación emocional.

De acuerdo con Yus (2014: 6-14), los emoticonos pueden desempeñar hasta ocho funciones distintas: 1. señalar la actitud proposicional que subyace al enunciado; 2. intensificar la actitud proposicional codificada verbalmente; 3 . intensificar o atenuar la fuerza ilocutiva de un acto de habla; 4. contradecir el contenido codificado verbalmente mediante bromas; 5 . contradecir el contenido codificado 
verbalmente mediante la ironía; 6. indicar una actitud afectiva hacia el contenido del mensaje; 7. indicar una actitud afectiva hacia el acto de habla; y 8. intensificar una actitud afectiva que ya se ha codificado verbalmente. En este sentido, los emoticonos que actúan como signos de puntuación no pierden su función actitudinal o emocional concreta, sino que enriquecen la interacción cohesionando las distintas unidades discursivas y guiando la interpretación de las emociones y actitudes, tanto si se encuentran codificadas verbalmente como si no.

Para que adquieran una función cohesiva, los emoticonos dependen mucho de la posición que ocupen en el mensaje. Estos pueden aparecer solos o acompañados de texto $\mathrm{y}$, dentro de estos últimos, al principio, en el medio o al final del mensaje, acompañándolo o sustituyendo un elemento léxico. Por tanto, el hecho de que un emoticono pueda actuar como un signo de puntuación no implica que siempre lo haga. Pueden ser signos de puntuación los que aparecen en el interior o al final de un mensaje con texto sin sustituir elementos léxicos delimitando unidades discursivas, pues estos se sitúan en los mismos lugares donde se situaría otro signo de puntuación que carece de valor retórico-emotivo.

(12) M1: Buenos días amigas. Me acabo de levantar después de una noche de sueños muy divertidos $\because$ Anoche cuando me acosté sentía la patatilla y no me gustaba, pero me he despertado bastante bien. Creo que este partido también lo he ganado. Hasta el martes vida reposada después de haber limpiado la casa (es mu madrugador) Gracias por estar ahí. Os quiero

En (12) se observa un caso muy claro de uso de los emoticonos como signos de puntuación y, de hecho, en este ejemplo la hablante alterna enunciados delimitados por puntos con enunciados delimitados por emoticonos. De esta forma, en lugar de un signo de puntuación convencional de primer orden, se utilizan elementos icónicos que, además de funcionar como elementos cohesivos, intensifican la actitud proposicional codificada verbalmente en el primer caso, actúan como elementos decorativos (Sampietro, 2016b) en el segundo e intensifican una actitud afectiva que ya se ha codificado verbalmente en el último caso. Además, muestra del uso consciente que está haciendo la hablante 
de los emoticonos como puntos, en este caso, es que sigue la norma de comenzar el siguiente enunciado con mayúscula.

Por último, cabe destacar que los emoticonos son utilizados como signos de puntuación con más frecuencia por los hablantes mayores, puesto que estos suelen sustituirse por signos de primer orden y son los hablantes de esta franja de edad los que más utilizan también el punto y la coma. Asimismo, pese a que solo se está teniendo en cuenta la variable de la edad por las características de la muestra recogida, se ha observado también un uso más abundante de los emoticonos como signos de puntuación por mujeres que por hombres. Sin embargo, para sacar conclusiones determinantes en este aspecto resulta necesario realizar en el futuro un estudio exhaustivo que permita tener en cuenta esta variable.

\section{3. Signos modalizadores}

Al contrario de lo que ocurre con los signos de primer orden, los modalizadores tienden a aumentar su uso. Se observan casos de ausencia del signo de interrogación en mensajes interrogativos, siempre en hablantes jóvenes. Esto puede deberse, por un lado, a la inmediatez y, por otro lado, a que el uso de pronombres interrogativos en estos enunciados no plantea ambigüedad para interpretar la modalidad del mensaje.

(13) a. M1: Qué tal mamá (WA03, 1, J)

b. M4: Como vas Bea (WA04, 53, J)

El corpus muestra cómo los signos de interrogación y de exclamación de cierre se repiten para dar énfasis al enunciado. Este recurso es más utilizado por los hablantes jóvenes. En (14a), por ejemplo, se utilizan tres signos de interrogación para mostrar insistencia en la pregunta y, en (14b), se utilizan cuatro signos de exclamación para intensificar el acto de habla de la felicitación. Los de apertura, por el contrario, tienden a no utilizarse; solo se observa su uso en algunos hablantes de mayor edad (15).

(14) a. M4: Cómo quedamos??? (WA04, 31, J)

b. M1: Feliz año nuevo chicas!!!! (WA04, 1, J)

(15) M2: ¿Hay que decidir si queremos cocido? (WA07, 13, M) 
En cambio, aunque hay casos de algún punto más de los tres reglamentarios en el uso de los puntos suspensivos, lo común es que se escriban solo tres (16).

(16) a. M2: Pues en mi casa..... (WA04, 67, J)

b. H1: A los que no han dicho nada les supongo absentistas, porque ya habrán reflexionado suficiente... (WA07, 2-3, M)

\section{4. Introductores de un segundo discurso}

En cuanto a los signos de segundo régimen que introducen un discurso secundario, estos apenas se utilizan. Destaca el uso de los paréntesis para delimitar los incisos, utilizados sobre todo por los hablantes de mayor edad (17).

(17) M1: Acabo de leer que nuestra Willie fock (o como se escriba) está en Barna $($ WA06, 39-40, M)

Sin embargo, llama la atención la escasa presencia de comillas, que aparecen en un solo mensaje, ejemplificado en (18), pese a la existencia de discursos reproducidos en el corpus y a su uso común en el habla coloquial.

(19) M1: ... Nivel de conversación: - "buenos días" - "Serán pa ti"를 ㅇํㅇ (WA06, 91-92, M)

Por último, cabe destacar que la raya no se documenta en todo el corpus, pues para desempeñar su función los hablantes muestran una clara preferencia por los paréntesis. Además, la raya junto con el punto y coma son los dos signos ausentes en el corpus. No obstante, hay que tener en cuenta que tanto las comillas como los dos puntos se documentan en un solo caso y en dos, respectivamente. 


\section{CONCLUSIONES}

Partiendo de la premisa de que los signos de puntuación codifican instrucciones de procesamiento, optimizando así la relevancia del texto, se ha concluido que los usos no normativos de la puntuación en los mensajes instantáneos de WhatsApp responden a una intención comunicativa del hablante. La necesidad de suplir las carencias contextuales de este tipo de interacciones mediadas ha llevado a desarrollar una puntuación retórico-emotiva donde la transmisión de información contextual prima sobre las relaciones lógicas entre las unidades. Así pues, se reduce el uso de los signos de primer orden manteniéndose el punto y la coma como únicos delimitadores- y aumenta la frecuencia y repetición de los modalizadores. Del mismo modo, los signos de primer orden, en algunos casos, son sustituidos por emoticonos que, a la par que indican instrucciones de procesamiento, guían la interpretación emocional del mensaje.

En este trabajo, teniendo en cuenta que existe una búsqueda de la relevancia óptima, se ha tratado de sistematizar los cambios que se están produciendo en la puntuación y de encontrarles una razón de ser. Del mismo modo que con la expansión de la lectura silenciosa se perdió el valor prosódico de la puntuación, con la revolución tecnológica se ha producido un cambio en las relaciones entre oralidad y escritura y se han desarrollado estrategias pragmático-discursivas que han afectado a la puntuación. Por tanto, como ya ha ocurrido a lo largo de la historia, en la actualidad se está produciendo una nueva normalización de la puntuación que afecta a los textos digitales. En este sentido, hay que señalar que la puntuación empleada en WhatsApp no es arbitraria, sino que depende de las condiciones concretas de enunciación del mensaje. Por ello, en este análisis también se han considerado relevantes los factores relacionados con el tipo de texto y sus condiciones relativas al registro, planificación y relación de proximidad, así como el dispositivo utilizado.

En cuanto a la edad, parece que esta sí influye en la selección de la puntuación en WhatsApp. Los datos obtenidos del corpus muestran que los hablantes mayores utilizan más puntuación, tanto normativa como retórico-emotiva. Llama la atención que estos hablantes, que son inmigrantes digitales y se han familiarizado con estos usos de la puntuación ya siendo adultos, emplean con especial frecuencia los usos retórico-emotivos que se han desarrollado en el contexto digital. En cambio, los jóvenes, que sí son nativos digitales, apenas utilizan 
puntuación, ni siquiera como un recurso retórico-emotivo. Esto puede que sea un indicador del camino que está siguiendo la puntuación en su proceso de normalización en estos textos y sería interesante observar su evolución, es decir, si se confirma la tendencia a la desaparición de la puntuación en WhatsApp de acuerdo a lo observado en los hablantes jóvenes o si se mantiene su uso como recurso retórico-emotivo.

En definitiva, en el presente estudio se ha atendido a las distintas funciones que se le han atribuido a la puntuación a lo largo de la historia para justificar la posibilidad de que esta haya adquirido una nueva función en los textos digitales. Asimismo, se han observado las particularidades de los textos digitales y, en concreto, de los mensajes instantáneos de WhatsApp que determinan la selección de la puntuación en una interacción mediada. Por último, tras un análisis cualicuantitativo de los datos obtenidos de un corpus, se ha concluido que los usos no normativos parecen responder a la intención de los hablantes de transmitir actitudes proposicionales y emociones en un tipo de interacción mediada, así como que existen grandes diferencias en el uso de la puntuación en función de la edad: los jóvenes apenas la utilizan y los mayores la utilizan de acuerdo con la norma y como un recurso retórico-emotivo.

\section{Bibliografía}

Alcoba, S. (2000). Puntuación y melodía de la frase. En Alcoba, S. (coord.), La expresión oral (147-186). Barcelona: Ariel.

Alcántara Plá, M. (2014). Las unidades discursivas en los mensajes instantáneos de wasap. En Estudios de Lingüística del Español, 35, 223-242.

Baron, N. S. (2010). Always On. Language in an Online and Mobile World. Nueva York: Oxford University Press.

Benito Lobo, J. A. (1992). La puntuación: usos y funciones. Madrid: Edinumen.

Briz Gómez, A. (1998). El español coloquial en la conversación: esbozo de pragmagramática. Barcelona: Ariel.

Cassany, D. (1987 [2001]). Describir el escribir, 10. a ed. Barcelona: Paidós.

Cassany, D. (1993). La cocina de la escritura. Barcelona: Anagrama, 1995. 
Cassany, D. (1999). Puntuación: investigaciones, concepciones y didáctica. En Letras, $58,21-54$.

Cassany, D. (2006). Tras las líneas. Sobre la lectura contemporánea. Barcelona: Anagrama.

Cassany, D. (2012). En_línea. Leer y escribir en la red. Barcelona: Anagrama.

Catach, N. (1980). La ponctuation. En Langue française, 45, 16-27. Disponible en: https://doi.org/10.3406/lfr.1980.5260, consulta: 10/04/2019.

Crystal, D. (2002). El lenguaje e Internet. Cambridge: Cambridge University Press. Traducción al español de Pedro Tena.

Crystal, D. (2005). La revolución del lenguaje. Madrid: Alianza. Traducción al español de Francisco Muñoz de Bustillo.

DPD = Real Academia Española y Asociación de Academias de la Lengua Española (2005). Diccionario panhispánico de dudas. Madrid: Santillana. Disponible en: http://www.rae.es/recursos/diccionarios/dpd.

Ferreiro, E. (1996). Los límites del discurso: puntuación y organización textual. En Ferreiro, E.; Pontecorvo, C.; Ribeiro, N. y García, I., Caperucita Roja aprende a escribir (129-161). Barcelona: Gedisa.

Figueras, C. (2001). Pragmática de la puntuación. Barcelona: EUB: Octaedro.

Figueras, C. (2014). Pragmática de la puntuación y nuevas tecnologías. En Normas, 4, 135-160.

Gómez Torrego, L. (2001). La gramática en Internet. En II Congreso Internacional de la Lengua Española. Nuevas fronteras del español: Lengua y escritura en Internet, Valladolid: Centro Virtual Cervantes. Disponible en: http://congresosdelalengua.es/valladolid/ponencias/nuevas_fronteras_del_espa nol/4_lengua_y_escritura/gomez_l.htm, consulta: 02/05/2019.

Gómez Torrego, L. (2006). Hablar y escribir correctamente. Gramática normativa del español actual. Madrid: Arco/Libros.

Halliday, M. A. K. (1990). Spoken and Witten Language. Oxford: Oxford University Press.

Herring, S. C. (2019). Grammar and Electronic Communication. En C. Chapelle (ed.), The Concise Encyclopedia of Applied Linguistics. Hoboken: Wiley-Blackwell. 
Jaffe, J. M.; Lee, Y.; Huang, L. y Oshagan, H. (1995). Gender, pseudonyms and CMC: Masking identities and baring souls. En 45th Annual Conference of the International Communication Association. Alburquerque, Nuevo México, EEUU. Disponible en: https://smg.media.mit.edu/library/jafe1995.html, consulta: 30/04/2019.

Linares, M. (1979). Estilística. Teoría de la puntuación. Ciencia del estilo lógico. Madrid: Paraninfo.

Mancera Rueda, A. (2016). Usos lingüísticos alejados del español normativo como seña de identidad en las redes sociales. En Bulletin os Spanish Studies, Vol. XCIII, n. ${ }^{\circ}$ 9, 1469-1493. Disponible en: http://dx.doi.org/10.1080/14753820.2016.1181435, consulta: 02/05/2019.

Markman, K. M. y Oshima, S. (2007). Pragmatic play? Some possible functions of English emoticons and Japanese Kaomoji in computer-mediated discourse. En Association of Interner Researchers Annual Conference 8.0: Let's play!, Vancouver, Canadá. Disponible en: https://www.academia.edu/2666102/Pragmatic_play_Some_possible_functions _of_English_emoticons and Japanese_kaomoji_in_computermediated discourse, consulta: 01/05/2019.

Martínez de Sousa, J. (2004). Ortografía y ortotipografía del español actual. Gijón: Trea.

Millán, J. A. (2005). Perdón imposible. Barcelona: RBA.

Moliner, M. (1967). Diccionario de uso del español. Barcelona: Gredos.

Montolío, E. (2001). Conectores de la lengua escrita. Barcelona: Ariel.

Nunberg, G. (1990). The Linguistics of Punctuation. Palo Alto: CSLI.

OLE = Real Academia Española y Asociación de Academias de la Lengua Española (2010). Ortografía de la lengua española. Madrid: Espasa Calpe.

Parkes, M. B. (1992). Pause and Effect. An Introduction to the History of Punctuation in the West. Aldershot (Hampshire): Scholar Press.

Penas Ibáñez, M. ${ }^{a}$ A. (2018). El cibertexto y el ciberlenguaje. Madrid: Síntesis.Polo, J. (1974). Ortografía y ciencia del lenguaje. Madrid: Paraninfo.

Polo, J. (1990). Manifiesto ortográfico de la lengua española. Madrid: Visor Libros.

Prensky, M. (2001). Digital Natives, Digital Immigrants. En On the Horizon, MCB University Press, Vol. 9, n. ${ }^{\circ}$ 5, 1-6. 
Real Academia Española (1969[1726-1737]). Diccionario de autoridades, Tomo I. Madrid: Gredos, 1726.

Real Academia Española (1741). Orthographía española. Madrid: Imprenta de la Real Academia Española.

Disponible en: $\quad$ http://www.rae.es/sites/default/files/Ortografia_RAE_1741_reducida.pdf, consulta: 25/04/2019.

Real Academia Española (1754). Ortografía de la lengua castellana. Disponible en: https://bivaldi.gva.es/es/consulta/registro.cmd?id=6648, consulta: 25/04/2019.

Real Academia Española (1999). Ortografía de la lengua española. Madrid: Espasa Calpe.

Real Academia Española (2018). Libro de estilo de la lengua española según la norma panhispánica. Barcelona: Planeta.

Sampietro, A. (2016a). Exploring the punctuation effect of emoji in Spanish whatsapp chats. En Lenguas Modernas, 47, 91-113.

Sampietro, A. (2016b). Emoticonos y emojis: Análisis de su historia, difusión y uso en la comunicación digital actual. Tesis doctoral, Valencia: Universitat de València.

Sánchez Iglesias, J. J. (2013). Puntuación y conexión. En Bustos Gisbert, J. M., Arquitextura fundamentos discursivos del texto escrito en español (137-155). Salamanca: Ediciones de la Universidad de Salamanca.

Santiago, R. (1996). La puntuación según Nebrija En DICENDA. Cuadernos de Filología Hispánica, ${ }^{\circ}$ 14, 273-284.

Santiago, R. (1998). Apuntes para la historia de la puntuación en los siglos XVI y XVII. En Blecua, J. M., Gutiérrez, J. y Sala, L. (eds.), Estudios de grafemática en el dominio hispánico (243-272) Salamanca: Ediciones Universidad de Salamanca.

Seco, M. (2011). Nuevo diccionario de dudas y dificultades de la lengua española. Barcelona: Espasa.

Serafini, M. T. (1992). Cómo se escribe. Barcelona: Paidós, 1994.

Sperber, D. y Wilson, D. (1986). Relevance. Communication and cognition. Oxford: Blackwell.

Torres i Vilatarsana, M. (2003). La llengua catalana en la comunicación a Internet: questió de codis. En Llengua i ús, n. ${ }^{\circ}$ 26, 77-82. Disponible en: https://core.ac.uk/download/pdf/39105902.pdf, consulta: 30/04/2019. 
Yus, F. (2001). Ciberpragmática. El uso del lenguaje en Internet. Barcelona: Ariel.

Yus, F. (2010). Ciberpragmática 2.0. Nuevos usos del lenguaje en Internet. Barcelona: Ariel.

Yus, F. (2014). Not all emoticons are created equal. En Linguagem em (Dis)curso, Vol. 14, n. ${ }^{\circ}$ 3, 511-529. Disponible en: http://dx.doi.org/10.1590/1982-4017-1403040414, consulta: 30/09/2019. 